\title{
Strange Dynamics in a Fractional Derivative of Complex-Order Network of Chaotic Oscillators
}

\author{
Carla M. A. Pinto
}

\begin{abstract}
We study the peculiar dynamical features of a fractional derivative of complex-order network. The network is composed of two unidirectional rings of cells, coupled through a "buffer" cell. The network has a $\mathbf{Z}_{3} \times \mathbf{Z}_{5}$ cyclic symmetry group. The complex derivative $D^{\alpha \pm \beta}$, with $\alpha, \beta \in \mathbf{R}^{+}$is a generalization of the concept of integer order derivative, where $\alpha=1, \beta=0$. Each cell is modeled by the Chen oscillator. Numerical simulations of the coupled cell system associated with the network expose patterns such as equilibria, periodic orbits, relaxation oscillations, quasiperiodic motion, and chaos, in one or in two rings of cells. In addition, fixing $\beta=0.8$, we perceive differences in the qualitative behavior of the system, as the parameter $c \in[13,24]$ of the Chen oscillator and/or the real part of the fractional derivative, $\alpha \in\{0.5,0.6,0.7,0.8,0.9,1.0\}$, are varied. Some patterns produced by the coupled system are constrained by the network architecture, but other features are only understood in the light of the internal dynamics of each cell, in this case, the Chen oscillator. What is more important, architecture and/or internal dynamics?
\end{abstract}

Keywords: Chaos; quasiperiodic motion; periodic solutions; Hopf bifurcation; period-doubling bifurcation; period-halving bifurcation; fractional derivative.

\section{Introduction}

Networks of coupled cells have been widely studied in the literature. The main reason is their applicability to the modeling of a large variety of real life phenomena, provided by their wide range of new and unexpected dynamical patterns. Examples are in gene transcription networks, animal and robot locomotion, ecological networks, economics, physics, the World Wide Web, secure communication, automatic control, social networks, neuroscience, secure communication, automatic control, neuroscience, and cryptography, to name a few.
Readers can refer to [Singer, 1999; Dachselt \& Schwarz, 2002; Albert \& Barabási, 2002; Xie et al., 2002; Boccaletti et al., 2006; Pinto \& Golubitsky, 2006; Pinto, 2012, 2014; Zhu et al., 2013].

A dynamically evolving network may exhibit distinct dynamical features, from synchronized states [Pikovsky et al., 2003; Stewart \& Parker, 2007; Aguiar et al., 2011], phase locking [Pikovsky et al., 2003], resonance, quasiperiodicity, and other complex patterns [Huxter et al., 2003; Ikegaya et al., 2004; Antoneli et al., 2010; Pinto, 2012]. Synchronized states are extremely important in daily life 
[Zhu et al., 2013]. The network architecture is greatly responsible for the appearance of these behavioral patterns [Terman et al., 2002; Wang, 2002; Stewart et al., 2003; Golubitsky et al., 2004; Vragović et al., 2006; Boccaletti et al., 2006]. For example, synchronized patterns, in which two or more nodes behave identically, and phase relations, in which the behavior is identical except for a fixed time delay, are expected in a network with a "symmetric" architecture. Numerical and experimental outcomes also confirm the existence of this relationship between network architecture and network dynamics [Prinz et al., 2004].

Stewart et al. [2003] and Golubitsky et al. [2005] developed a theory of coupled cell networks and associated dynamical systems. A network of coupled cells may be realized through a directed graph, where the nodes represent the cells and the arrows the couplings between them. A "cell" is commonly defined as a system of ordinary differential equations. The dynamics of the full network consists of the dynamics of the time evolution at all the nodes of the network. This formalism frees the researcher of the details of the dynamics of each node and allows him to focus only on the network structure. Nevertheless, as was pointed before by Antoneli et al. [2010], Pinto [2012, 2014], there are exotic features that cannot be explained solely by the network architecture and seem to be explained by the internal dynamics of each node (or cell).

Bearing these ideas in mind, in this paper, we study the complex-order network of two unidirectional rings of cells, coupled through a "buffer" cell, with $\mathbf{Z}_{3} \times \mathbf{Z}_{5}$ symmetry (Fig. 1).

In Sec. 2, we revise briefly the coupled cells networks formalism and bifurcation theory. In Sec. 3, we simulate the fractional complex-order coupled cell system associated with the network in Fig. 1 and discuss the implications of the results. In Sec. 4,

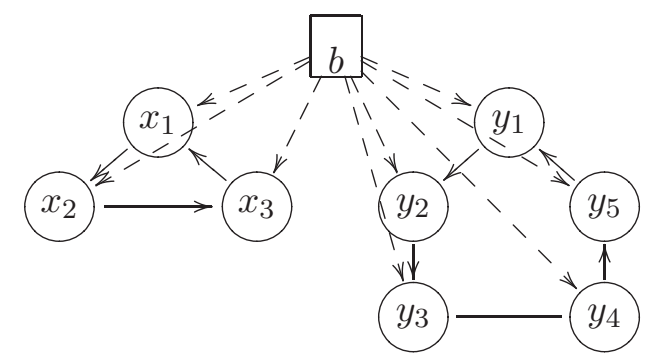

Fig. 1. Network of two coupled unidirectional rings, one with three cells and the other with five, connected through a buffer cell $b$. The network has $\mathbf{Z}_{3} \times \mathbf{Z}_{5}$ symmetry group. we conclude this work and shed some light for future research directions.

\subsection{Fractional derivative of complex-order}

Fractional calculus (FC) is a generalization of the integer order differentiation and integration to an arbitrary, real or complex order [Podlubny, 1998; Kilbas et al., 2006]. Several applications have emerged in engineering, physics, biology, fluid mechanics, electrochemistry, and many other research areas [Tenreiro Machado, 1997; Mainardi, 2010; Tarasov, 2010; Oldham, 2010; Pinto \& Machado, 2011a, 2011b, 2014; Li \& Ma, 2013].

There are three main definitions of fractional derivatives of order $\alpha \in \mathbf{R}$, namely, the RiemannLiouville, the Grünwald-Letnikov, and the Caputo, given by:

$$
\begin{gathered}
{ }_{a}^{\mathrm{RL}} D_{t}^{\alpha} f(t)=\frac{1}{\Gamma(n-\alpha)} \frac{d^{n}}{d t^{n}} \int_{a}^{t} \frac{f(\tau)}{(t-\tau)^{\alpha-n+1}} d \tau, \\
t>a, \quad \operatorname{Re}(\alpha) \in] n-1, n[ \\
{ }_{a}^{\mathrm{GL}} D_{t}^{\alpha} f(t)=\lim _{h \rightarrow 0} \frac{1}{h^{\alpha}} \sum_{k=0}^{\left[\frac{t-a}{h}\right]}(-1)^{k}\left(\begin{array}{l}
\alpha \\
k
\end{array}\right) f(t-k h), \\
t>a, \quad \alpha>0 \\
{ }_{a}^{\mathrm{C}} D_{t}^{\alpha} f(t)=\frac{1}{\Gamma(n-\alpha)} \int_{a}^{t} \frac{f^{(n)}(\tau)}{(t-\tau)^{\alpha-n+1}} d \tau, \\
t>a, \quad n-1<\alpha<n
\end{gathered}
$$

where $\Gamma(\cdot)$ is Euler's gamma function, $[x]$ means the integer part of $x$, and $h$ is the step time increment.

The fractional derivatives have memory, in the sense that they "learn" with the history of the past dynamics of the system. Integer order systems are, in opposition, considered "local" operators.

The Grünwald-Letnikov formulation inspires the numerical calculation of the fractional derivative based on the approximation of the time increment $h$ through the sampling period $T$ and the series truncation at the $r$ th term. This method is often denoted as Power Series Expansion (PSE) yielding the equation in the $z$-domain:

$$
\frac{\mathcal{Z}\left\{D^{\alpha} f(t)\right\}}{\mathcal{Z}\{f(t)\}}=\frac{1}{T^{\alpha}} \sum_{k=0}^{r} \frac{(-1)^{k} \Gamma(\alpha+1)}{k ! \Gamma(\alpha-k+1)} z^{-k}
$$

where, in order to have good approximations, a large $r$ is required and a small value of $T$. 
Expression (4) represents the Euler, or first backward difference, approximation in the so-called $s \rightarrow z$ conversion scheme.

The most known generalized derivative operator considers $\alpha \in \mathbf{R}$. The fractional derivative of complex-order $\alpha \pm \jmath \beta \in \mathbf{C}$ leads to complex output valued results. In [Hartley et al., 2005], the association of two complex-order derivatives is proposed, in order to overcome the complex results. In fact, there are several arrangements that produce real valued results. For example, with the real part of two complex conjugate derivatives $D^{\alpha \pm \jmath \beta}$ we get:

$$
\begin{aligned}
& Z\left\{\frac{1}{2}\left[D^{\alpha-j \beta} x(t)+D^{\alpha+j \beta} x(t)\right]\right\} \\
& \approx \frac{1}{T^{\alpha}}\left\{\sin \left[\beta \ln \left(\frac{1}{T}\right)\right]\right. \\
& \times\left[\beta z^{-1}+\frac{1}{2} \beta(1-2 \alpha) z^{-2}+\cdots\right. \\
&+\cos \left[\beta \ln \left(\frac{1}{T}\right)\right]\left[-1+\alpha z^{-1}\right. \\
&\left.\left.-\frac{1}{2} \beta\left(\alpha^{2}-\alpha-\beta^{2}+\cdots\right)\right]\right\} X(z) .
\end{aligned}
$$

We consider here expression (5) to perform the numerical simulations.

\section{Networks of Coupled Cell Systems}

A coupled cell network is schematically represented by a directed graph, with a finite set of nodes, representing the cells, and whose directed edges specify the coupling between them. The input set of a cell $c$ is the set of arrows that terminate at cell $c$. Two cells are input equivalent if there exists a bijection, between the corresponding input sets, that preserves the coupling type between the cells. Equivalent arrows have heads and tails of the same type, the equivalence relation on arrows is represented by $\simeq_{E}$. The equivalent relation on cells is symbolized by $\simeq_{C}$.

For each cell $c$ an internal phase space $P_{c}$ is defined. The total phase space of the network is the product $P=\prod_{i=1}^{n} P_{c}$. The coordinates on $P_{c}$ are denoted by $x_{c}$, the coordinates on $P$ are thus $\left(x_{1}, x_{2}, \ldots, x_{n}\right)$. At time $t$, the system is at state $\left(x_{1}(t), x_{2}(t), \ldots, x_{n}(t)\right)$.
A vector field $f$ on $P$, compatible with the network architecture, is said to be admissible for that network. Moreover, it satisfies the following two conditions: (1) the domain - each component $f_{i}$ corresponding to cell $c_{i}$ must be a function of the cells in the input set of cell $c_{i}, \mathcal{I}\left(c_{i}\right)$-; and (2) the pull-back condition - the components $f_{i}$ and $f_{j}$ of cells $c_{i}$ and $c_{j}$ are identical, up to a suitable permutation of the relevant variables, if the two cells have isomorphic input cells [Golubitsky \& Stewart, 2006].

The symmetry of a network imposes constraints on its dynamical behavior, and helps to explain some patterns that seem to be peculiar from the general coupled dynamical systems theory.

A symmetry of a coupled cells system is the group of permutations of the cells (and arrows) that preserve the network structure. This means that cells and arrows are permuted but cell-types and arrow-types are preserved. The action of the symmetry on $P$ is by permutation of the cell coordinates. The network in Fig. 1 is an example of a network with $\mathbf{Z}_{3} \times \mathbf{Z}_{5}$ symmetry. That means that we can permute the cells in the 3-ring and additionally we can exchange the cells in the 5 -ring.

\subsection{Bifurcations in symmetrically coupled cell systems - A review}

Golubitsky et al. [1988], Golubitsky and Stewart [2002] have developed a theory of local bifurcations of generic coupled cells systems with symmetry. The Equivariant Branching lemma gives sufficient conditions for the occurrence of a "symmetry-breaking" bifurcation from a fully symmetric equilibrium. Moreover, the Equivariant Hopf theorem guarantees the existence of branches of periodic solutions bifurcating from a fully symmetric equilibrium. We note that the bifurcating solutions have less symmetry than the fully symmetric equilibrium, the original solution.

The complete list of all spatiotemporal symmetries, produced by an integer order coupled cell system, associated with the network in Fig. 1, to be expected after a Hopf bifurcation, is determined in [Antoneli et al., 2010; Pinto, 2012, 2014]. We expect the same type of solutions for the fractional derivative of complex-order coupled cell system (8), associated with the network in Fig. 1.

The form of the periodic solutions that can be obtained when a coupled cell system associated 
with the network in Fig. 1 undergoes a primary Hopf bifurcation is described as follows. The periodic solution of type $\tilde{\mathbf{Z}}_{3} \times \mathbf{Z}_{5}$ is such that its components corresponding to the cells in the 3-ring are periodic and have the same wave form but they are $1 / 3$ out of phase and the components corresponding to the cells in the 5 -ring stay at equilibrium. Analogously, the periodic solution of type $\mathbf{Z}_{3} \times \tilde{\mathbf{Z}}_{5}$ is such that its components corresponding to the cells in the 5-ring are periodic and have the same wave form but they are $1 / 5$ out of phase, and the components corresponding to the cells in the 3-ring depict an equilibrium.

\section{Numerical Results}

We simulate the coupled cell system, associated with the network depicted in Fig. 1. We consider the Chen oscillator as the phase space for each cell of the two rings and an unidimensional phase space for the "buffer" cell. The total phase space is thus 25th-dimensional. The dynamics of a singular ring cell is given by Chen and Ueta [1999], Lü et al. [2002]:

$$
\begin{aligned}
\dot{u} & =a(v-u) \\
\dot{v} & =(c-a) u-u w+c v \\
\dot{w} & =u v-b w
\end{aligned}
$$

where $a=35, b=3, c$ are real parameters.

The unidimensional dynamics of the "buffer" cell is given by Antoneli et al. [2010]:

$$
f(u)=\mu u-\frac{1}{10} u^{2}-u^{3}
$$

where $\mu=-1.0$ is a real parameter.

The fractional derivative of complex-order coupled cells system of equations associated with the network in Fig. 1 is given by:

$$
\begin{aligned}
& \frac{1}{2}\left(D^{\alpha+\jmath \beta}+D^{\alpha-\jmath \beta}\right) x_{j} \\
& \quad=g\left(x_{j}\right)+k\left(x_{j}-x_{j+1}\right)+d b, \quad j=1, \ldots, 3 \\
& \frac{1}{2}\left(D^{\alpha+\jmath \beta}+D^{\alpha-\jmath \beta}\right) b=f(b), \\
& \frac{1}{2}\left(D^{\alpha+\jmath \beta}+D^{\alpha-\jmath \beta}\right) y_{j} \\
& \quad=g\left(y_{j}\right)+k\left(y_{j}-y_{j+1}\right)+d b, \quad j=1, \ldots, 5
\end{aligned}
$$

where $g(u)$ represents the dynamics of each Chen oscillator, $k=-5.0, d=0.2$, and the indexing assumes $x_{4} \equiv x_{1}$ and $y_{6} \equiv y_{1}$.

We fix $\beta=0.8$. We increase the value of the parameter $c \in[13,24]$, starting at $c=13$ from a fully equilibrium state in the cells of the two rings, for $\alpha=1.0$. For each value of $c$, we vary the real part of the fractional derivative of complex-order, $\alpha \in$ $\{0.5,0.6,0.7,0.8,0.9,1.0\}$, and study the dynamical behavior of the coupled cell system (8).

In Fig. 2, we plot the time series solution of the first variable $u$ of each Chen oscillator of the 3-ring (a) and the first variable $u$ of each Chen oscillator of the 5-ring (b), of the fractional derivative of complex-order coupled cell system (8). The cells in the 3-ring are at equilibrium and the cells in the 5ring depict a rotating wave state, for $\alpha=1.0$. Since we have started at a full equilibrium of the network, for $c=13$, the rotating wave state in the cells of the 5-ring appear after a primary Hopf bifurcation in these cells.

In Fig. 3, we plot the dynamics of the cells $x_{1}$ and $y_{1}$ of the two rings, for different values of $\alpha \in\{0.5,0.9,1.0\}$. We remark that we only plot the dynamics for these values of $\alpha$ for a better visualization, but the numerical simulations were performed for all values of $\alpha \in\{0.5,0.6,0.7,0.8,0.9,1.0\}$. Distinct dynamical behaviors can be seen in the cells of the two rings. In particular, rotating waves are exhibited by the cells in the 3-ring for $\alpha \in\{0.5$, $0.6,0.7,0.8,0.9\}$, and equilibrium is observed for $\alpha=1.0$. This suggests that a primary Hopf bifurcation has occured in the 3-ring for a certain value of $\alpha \in[0.9,1.0]$. Moreover, the cells in the 5-ring, show a rotating wave state for all values of $\alpha$. In addition, we remark that there is a decrease in the period and in the amplitude of the periodic solutions as $\alpha$ increases towards one. In fact, the rotating wave states in the cells of the 5-ring, for $\alpha=0.5$, already reveal a relaxation oscillation phenomena [Krupa \& Szmolyan, 2001].

Relaxation oscillations are solutions characterized by long periods of quasi-static behavior interspersed with short periods of rapid transition, commonly seen in fast-slow systems [Krupa \& Szmolyan, 2001].

In Fig. 4, the cells in the 3-ring and the cells in the 5-ring show a rotating wave state, for $\alpha=1.0$. A secondary Hopf bifurcation has occured in the 3-ring, as $c$ is increased from 13.7 till 13.9. 


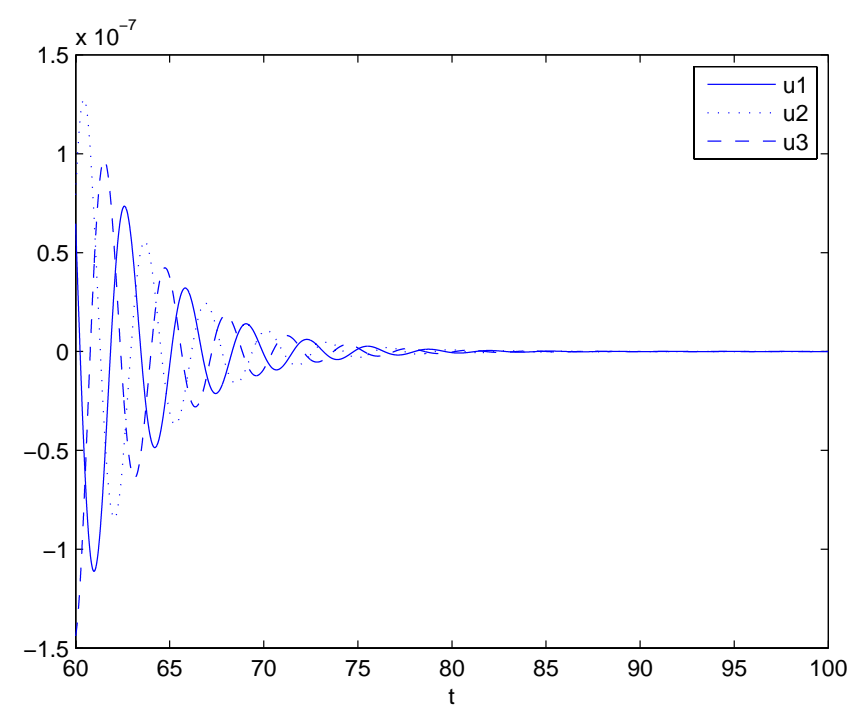

(a)

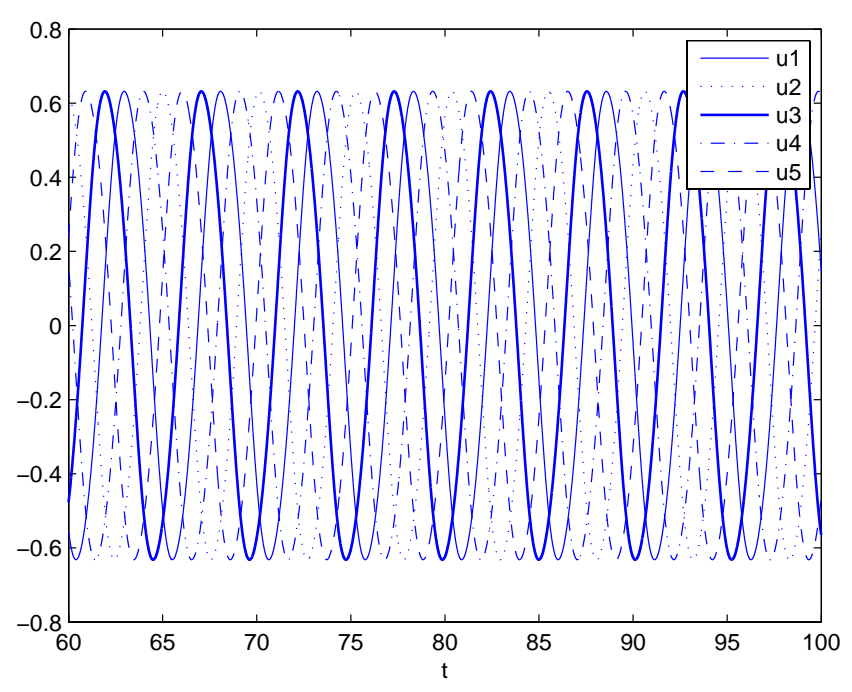

(b)

Fig. 2. Simulation results of the coupled cell system (8), for $a=35.0, b=3.0, c=13.7$ and $\alpha=1.0$. The cells in the 3-ring are at equilibrium. The cells in the 5 -ring depict a rotating wave state. Time series of the first variable $u$ of each Chen oscillator of (a) 3-ring and (b) 5-ring.

In Fig. 5, we plot the dynamics of the cells in the two rings, for different values of $\alpha \in$ $\{0.5,0.6,0.7,0.8,0.9,1.0\}$. We observe that, analogously to what happened for $c=13.7$, the period and the amplitude of the periodic solutions in the two rings decrease as $\alpha$ is increased towards one. In addition, relaxation oscillation behavior seems to appear in the cells of the 5 -ring for $\alpha \in\{0.5,0.6\}$.
Increasing further the parameter $c$, we obtain the behavior seen in Fig. 6. The cells in the 3-ring show a rotating wave state, whereas the cells in the 5 -ring depict a quasiperiodic motion, for $\alpha=1.0$.

In Fig. 7, we show the phase space plot of the first Chen oscillator in each ring of cells, for $\alpha=0.9$. Simulations have been performed for $\alpha \in\{0.5,0.6,0.7,0.8,0.9,1.0\}$. We observe that

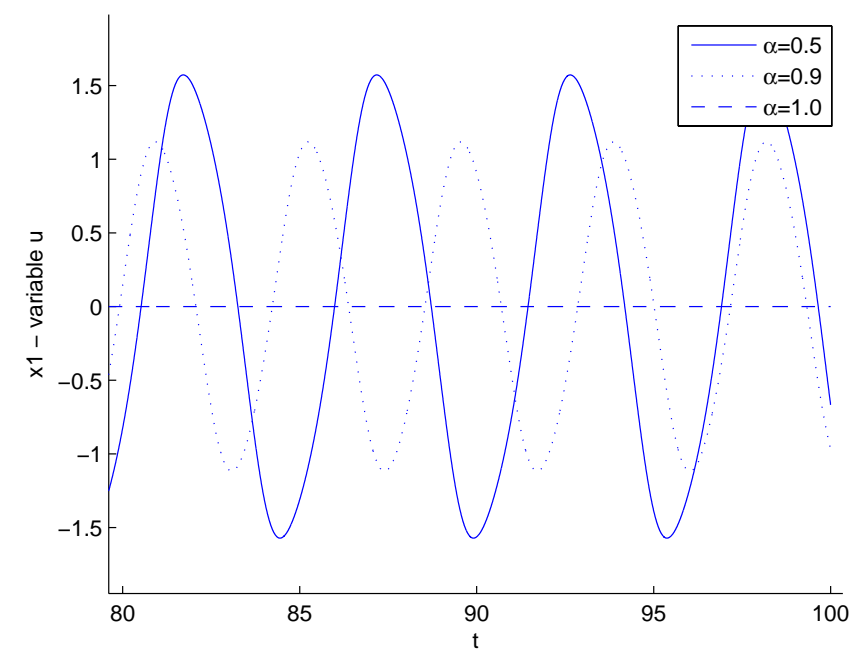

(a)

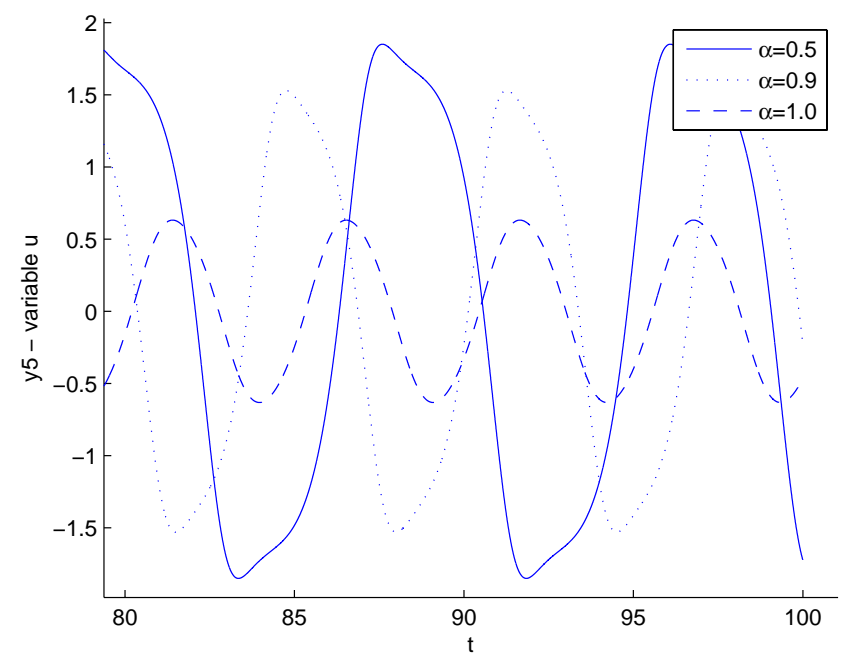

(b)

Fig. 3. Simulation results of the coupled cell system (8), for $a=35.0, b=3.0, c=13.7$, and distinct values of $\alpha \in$ $\{0.5,0.9,1.0\}$. The cells in the 3 -ring depict a rotating wave state for $\alpha \in\{0.5,0.6,0.7,0.8,0.9\}$ and are at equilibrium for $\alpha=1.0$. The cells in the 5 -ring show a rotating wave state for all values of $\alpha$. For more information, see text. Time series of the first variable of the Chen oscillator: (a) $x_{1}$ of the 3 -ring and (b) $y_{1}$ of the 5 -ring. 


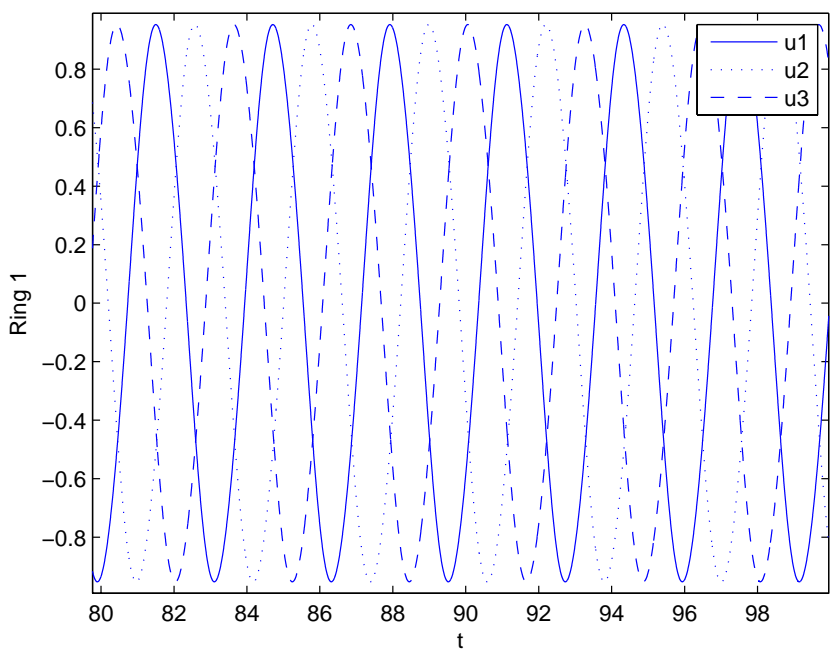

(a)

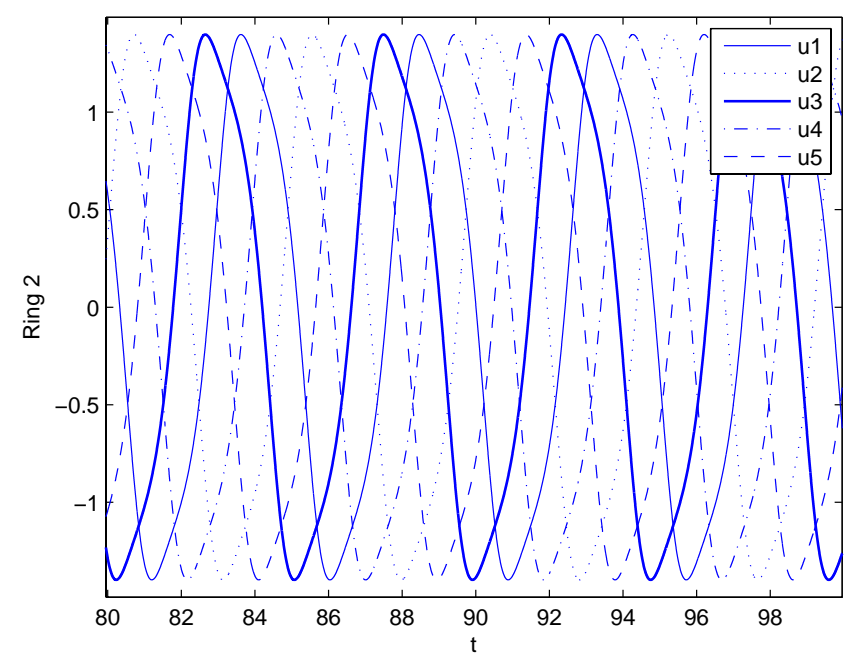

(b)

Fig. 4. Simulation results of the coupled cell system (8), for $a=35.0, b=3.0, c=13.9$ and $\alpha=1$. The cells in the two rings depict a rotating wave state. Time series of the first variable $u$ of each Chen oscillator of (a) 3-ring and (b) 5-ring.

the cells in the two rings depict a rotating wave for $\alpha=\{0.7,0.8,0.9\}$ and are at equilibrium for $\alpha=\{0.5,0.6\}$.

Continuing to vary the parameter $c$, the dynamical behavior of the coupled cell system (8) changes once more. In Fig. 8, we depict the phase space plots of the first Chen oscillator of each ring of cells and verify a quasiperiodic state in the 3-ring and a chaotic state in the 5 -ring, for $\alpha=1.0$.

In Figs. 9 and 10, the behavior of the cells is depicted in the two rings, for different values of $\alpha$. From the observation of the figures, we can conclude that, for $\alpha=\{0.5,0.6,0.7,0.8\}$, the cells in the two rings are at equilibrium. For $\alpha=0.9$, the cells in the two rings show a periodic solution of large period. Moreover, for $\alpha=1$, the cells in the 3-ring depict a quasiperiodic motion, whereas the cells in the 5-ring are at a chaotic state (see Fig. 8).

When increasing further the parameter $c$, another peculiar feature of the coupled cell system (8) emerges. In Fig. 11, we depict the phase space plots of the first Chen oscillator of each ring. The cells in the two rings show a chaotic behavior, for $\alpha=1.0$.

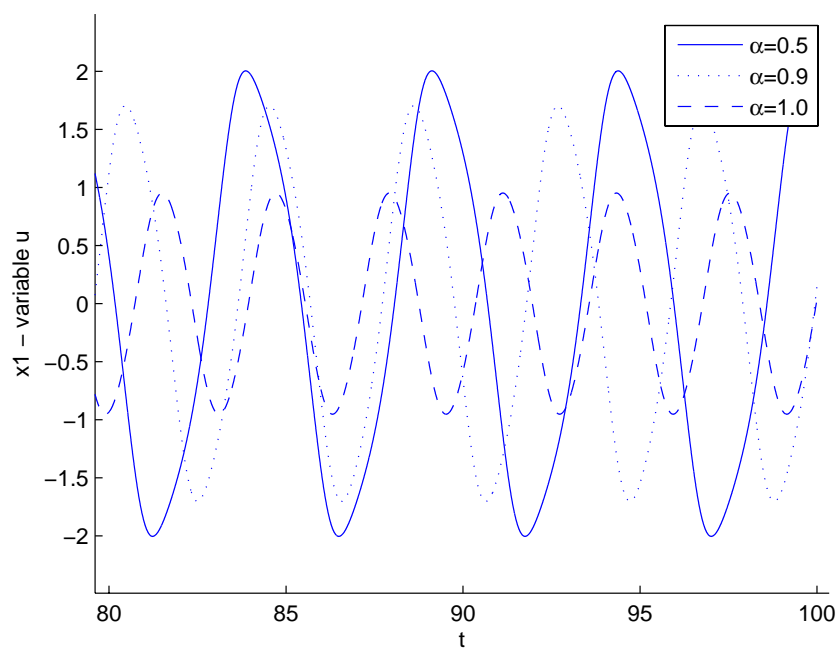

(a)

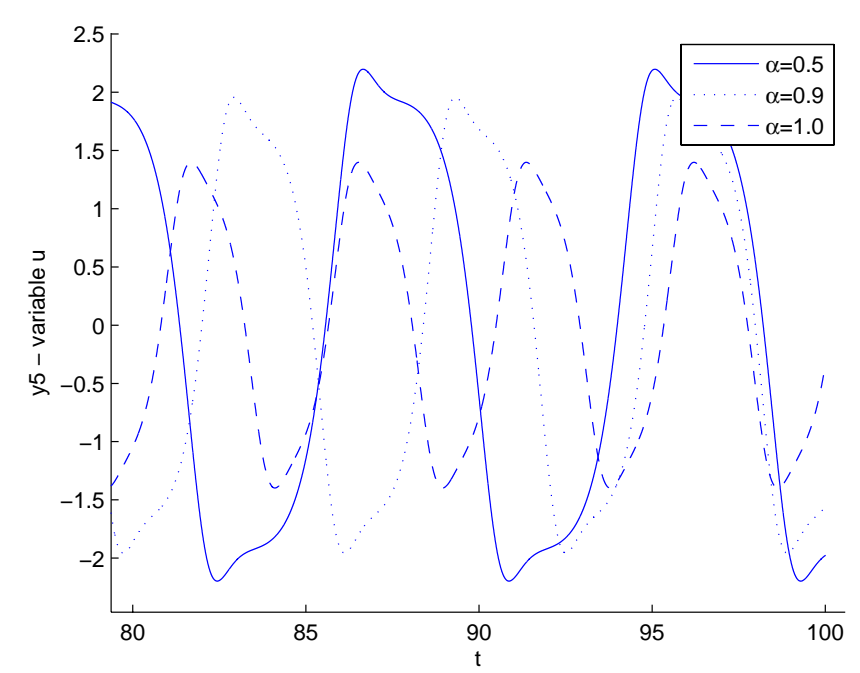

(b)

Fig. 5. Simulation results of the coupled cell system (8), for $a=35.0, b=3.0, c=13.9$, and $\alpha \in\{0.5,0.9,1.0\}$. The cells in the two rings show a rotating wave state for all values of $\alpha \in\{0.5,0.6,0.7,0.8,0.9,1.0\}$. For more information, see text. Time series of the first variable of the Chen oscillator: (a) $x_{1}$ of the 3 -ring and (b) $y_{1}$ in the 5 -ring. 


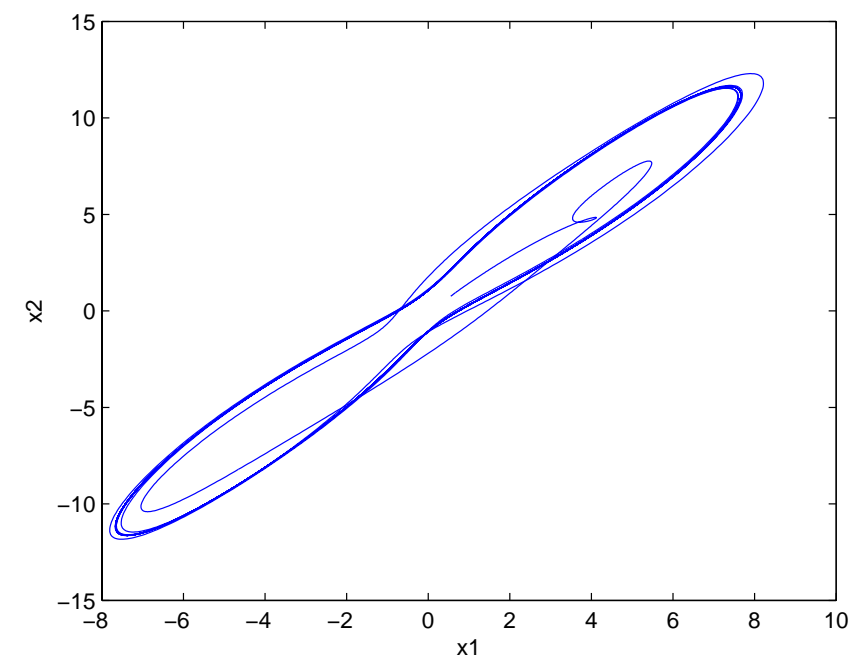

(a)

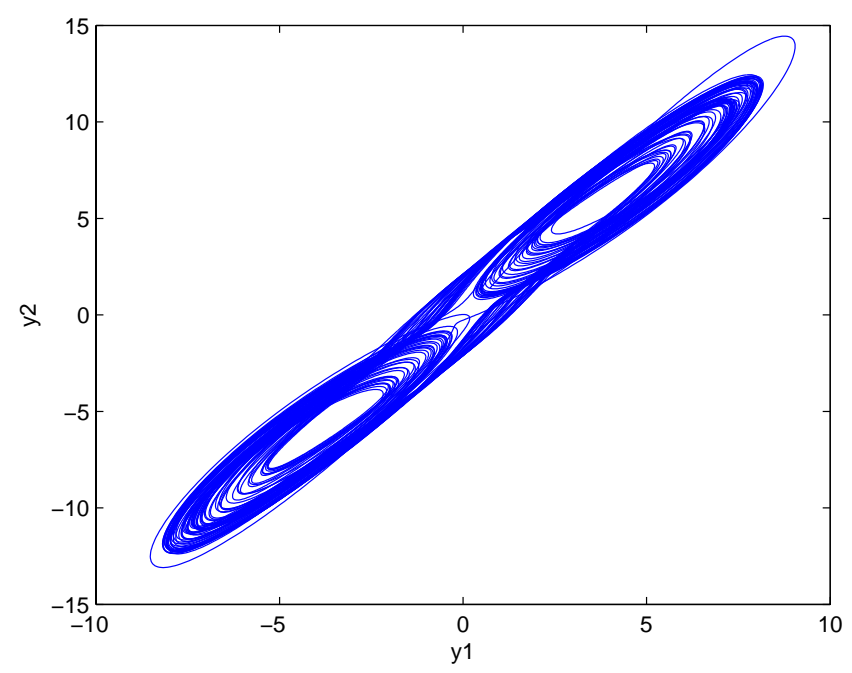

(b)

Fig. 6. Simulation results of the coupled cell system (8), for $a=35.0, b=3.0, c=17.5$ and $\alpha=1$. The cells in the 3-ring show a rotating wave state, whereas the cells in the 5-ring depict a quasiperiodic motion. Phase space plot of the first Chen oscillator of (a) 3-ring and (b) 5-ring.

The qualitative change in the dynamical behavior of system (8) seen in Figs. 6, 8 and 11, insinuates a cascade of period-doubling bifurcations leading the system from order (periodic solutions) to chaos, for $\alpha=1$.

Figure 12 shows a "simpler" pattern of the coupled cell system (8), for $c=21$ and $\alpha=0.9$, where the cells in the 3 -ring appear to be at a periodic state and cells in the 5-ring depict a quasiperiodic motion. Numerical simulations of the coupled system (8) for $\alpha=\{0.5,0.6,0.7,0.8,0.9,1.0\}$ and

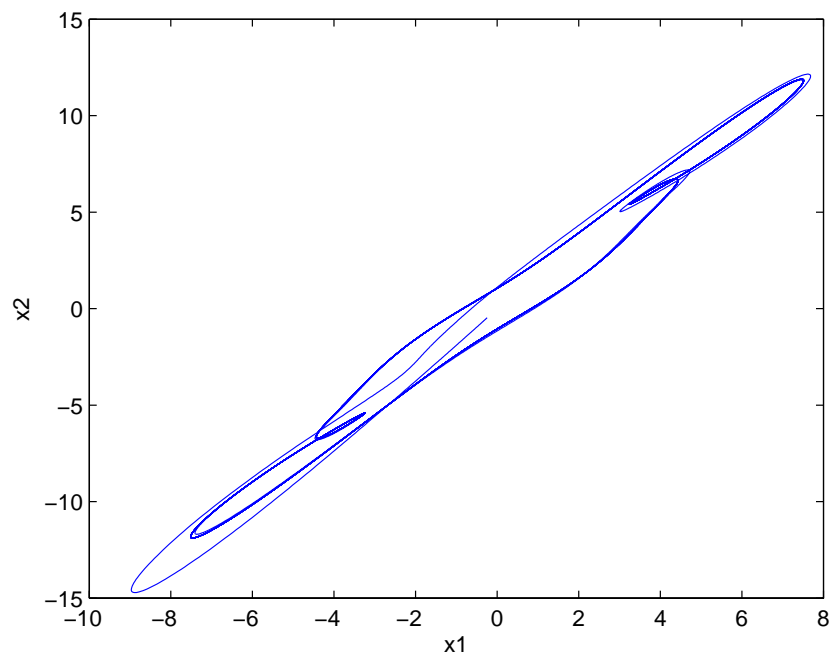

(a) $c=21$, unwrap even "simpler" patterns, for $\alpha=$ $\{0.5,0.6,0.7,0.8\}$, where the cells in the 3-ring are at equilibrium. Moreover, the cells in the 5-ring are at a periodic of "long period" state, for $\alpha=0.8$, whereas for $\alpha=\{0.5,0.6,0.7\}$ these cells are at equilibrium.

We continue increasing parameter $c$, and we distinguish a different dynamical behavior of the coupled cell system (8). In Fig. 13, we depict the time series of the cells in the two rings, for $\alpha=1.0$. Rotating wave states appear again in the cells of

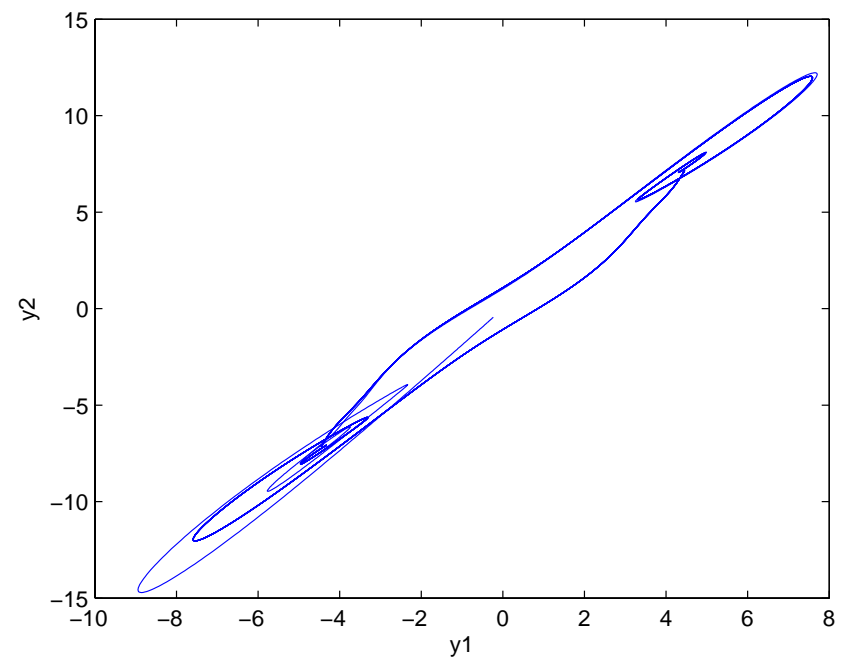

(b)

Fig. 7. Simulation results of the coupled cell system (8), for $a=35.0, b=3.0, c=17.5$ and $\alpha=0.9$. The cells in the two rings depict a rotating wave for $\alpha=\{0.7,0.8,0.9\}$ and are at equilibrium for $\alpha=\{0.5,0.6\}$. Phase space plot of the first Chen oscillator: (a) $x_{1}$ of 3-ring and (b) $y_{1}$ of 5-ring. 


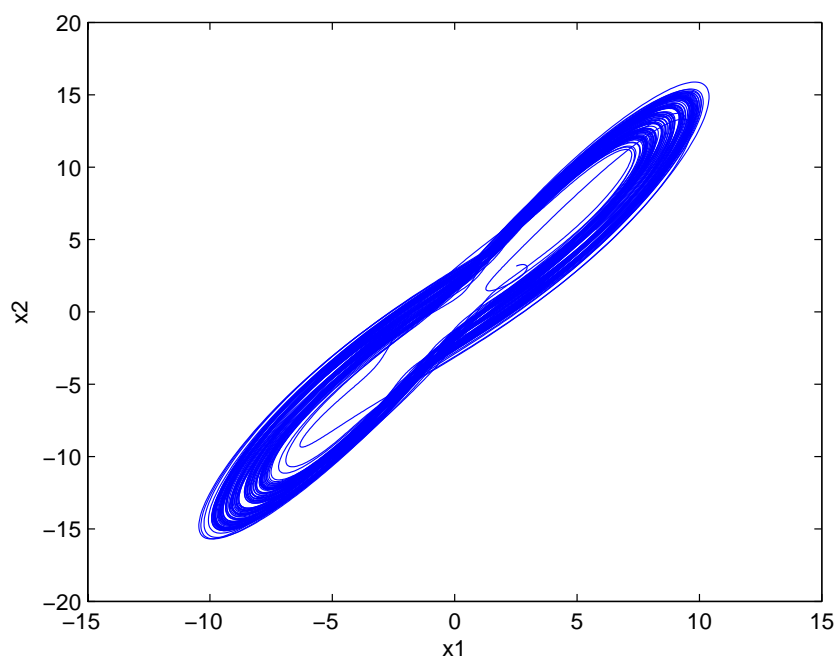

(a)

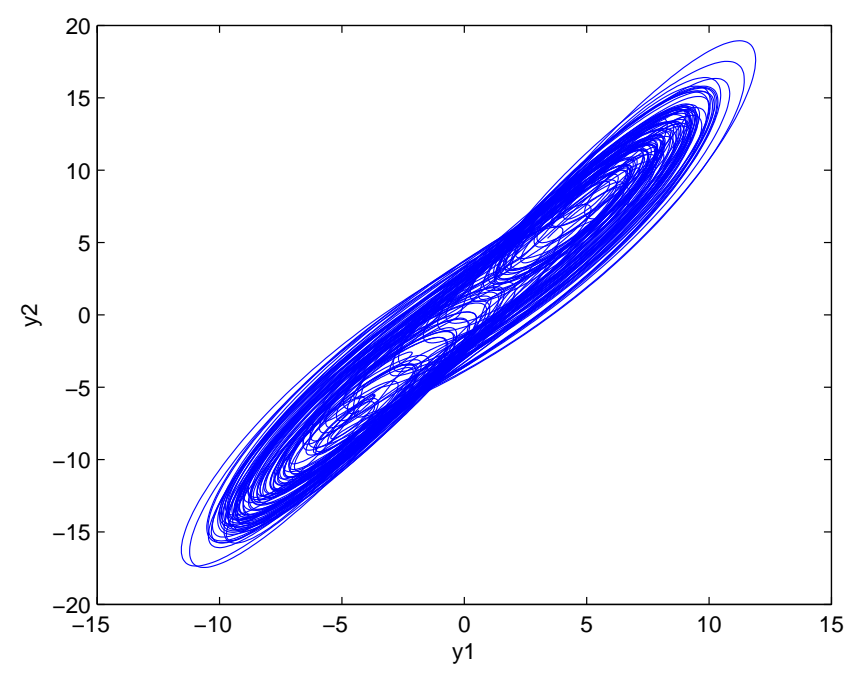

(b)

Fig. 8. Simulation results of the coupled cell system (8), for $a=35.0, b=3.0, c=19$ and $\alpha=1$. The cells in the 3-ring show a quasiperiodic motion, whereas the cells in the 5-ring are at a chaotic state. Phase space plot of the first Chen oscillator of (a) 3-ring and (b) 5-ring.

both rings. The periodic solution is clearly seen in Fig. 14. This change in the qualitative behavior of the coupled cell system (8) suggests the existence of a sequence of period-halving bifurcations that brings back the coupled cell system (8) from chaos to order (a stable periodic solution).

In Fig. 15, we plot the phase space of the coupled system (8) for $\alpha=0.9$. The cells in the two rings exhibit chaotic motions for this value of $\alpha$. Moreover, quasiperiodic motion in the cells of the

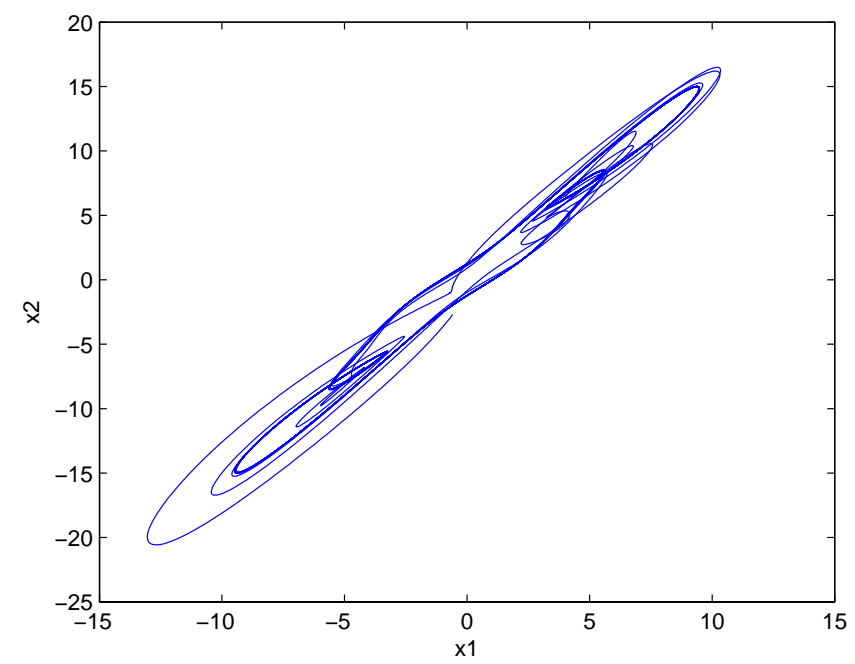

(a) two rings is seen for $\alpha=\{0.7,0.8\}$, and equilibrium for $\alpha=\{0.5,0.6\}$.

The simulations of the coupled cell system (8) uncover a very rich and complex dynamical behavior. In this sense, some considerations must be made:

(i) There is a change in the qualitative behavior of the coupled cell system (8) as $\alpha$, the real part of the fractional derivative of complex-order,

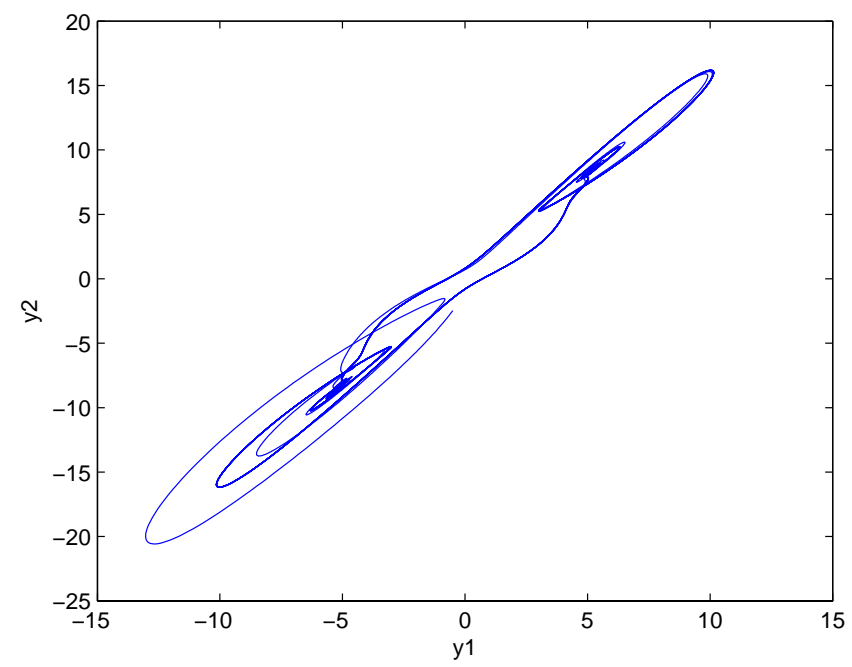

(b)

Fig. 9. Simulation results of the coupled cell system (8), for $a=35.0, b=3.0, c=19$ and $\alpha=0.9$. The cells in the two rings show a periodic orbit. Phase space plot of the first Chen oscillator of (a) 3-ring and (b) 5-ring. 


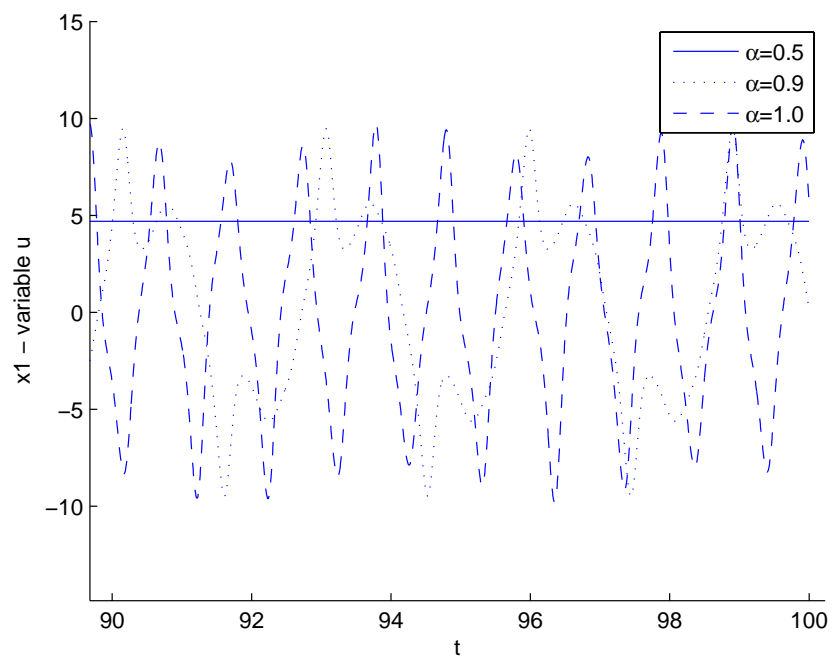

(a)

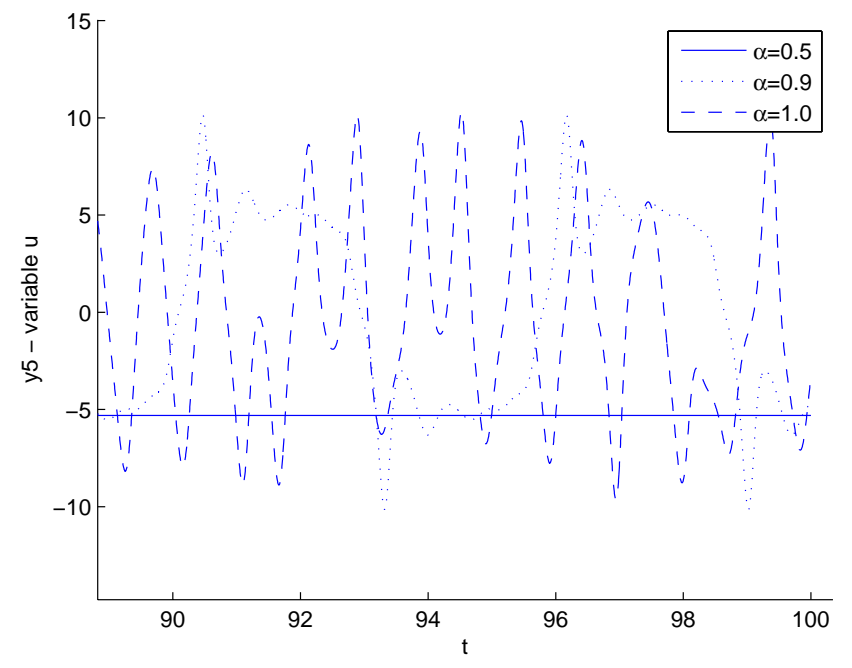

(b)

Fig. 10. Simulation results of the coupled cell system (8), for $a=35.0, b=3.0, c=19$ and $\alpha=\{0.5,0.9,1.0\}$. For $\alpha=\{0.5,0.6,0.7,0.8\}$, the cells in the two rings are at equilibrium. For $\alpha=0.9$, the cells in the two rings show a periodic solution. For $\alpha=1$, the cells in the 3-ring depict a quasiperiodic motion, whereas the cells in the 5-ring are at a chaotic state. Time series of the first variable of the Chen oscillator: (a) $x_{1}$ in 3 -ring and (b) $y_{1}$ in 5 -ring.

is varied. Thus, $\alpha$ plays here the role of a bifurcation parameter. Similar behavior is seen for variation of the parameter $c$.

(ii) Several Hopf bifurcations occur for variation of the parameter $c$ and for variation of $\alpha$.

(iii) Relaxation oscillations seem to be obtained, for $\alpha=1.0$, after a sequence of Hopf bifurcations. There is numerical evidence for, at least, two Hopf bifurcations, for increasing values of $c$. This behavior has been studied in

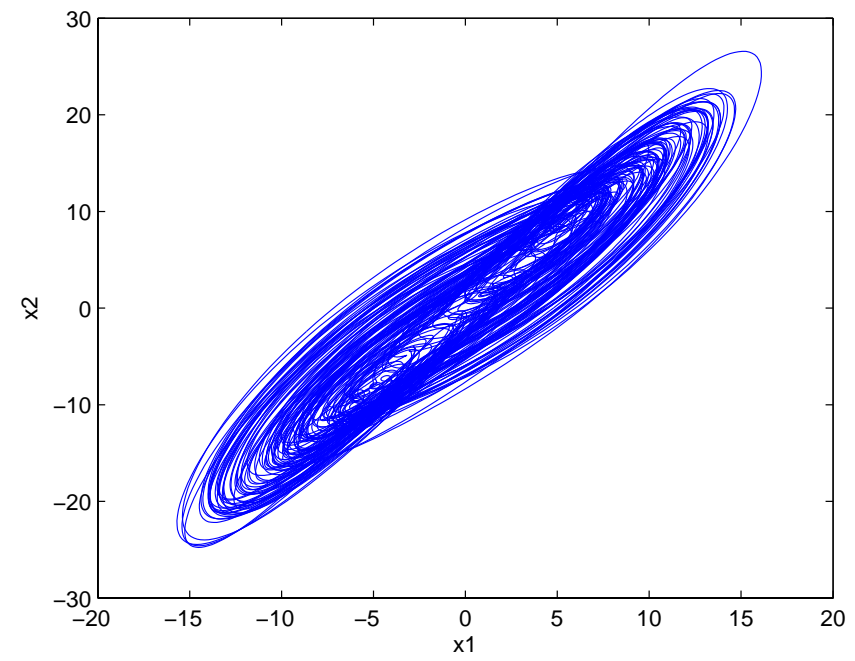

(a)
[Golubitsky et al., 2004; Antoneli et al., 2010; Pinto, 2012], where relaxation oscillation phenomena appear after a sequence of three Hopf bifurcations in an integer order coupled cell system, associated with the network in Fig. 1.

(iv) From the numerical results and from the already known behavior of integer order similar systems, we may suspect the existence of a cascade of Hopf bifurcations, period-doubling bifurcations and period-halving bifurcations.

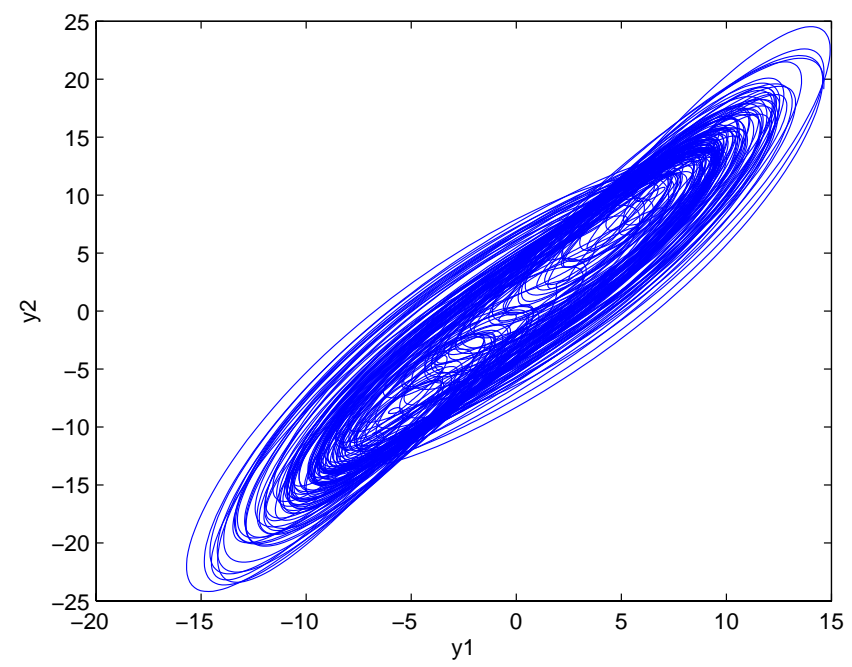

(b)

Fig. 11. Simulation results of the coupled cell system (8), for $a=35.0, b=3.0, c=21$ and $\alpha=1$. The cells in the 3-ring show quasiperiodic motion, and the cells in the 5-ring depict a chaotic behavior. Phase space plot of the first Chen oscillator of (a) 3-ring and (b) 5-ring. 


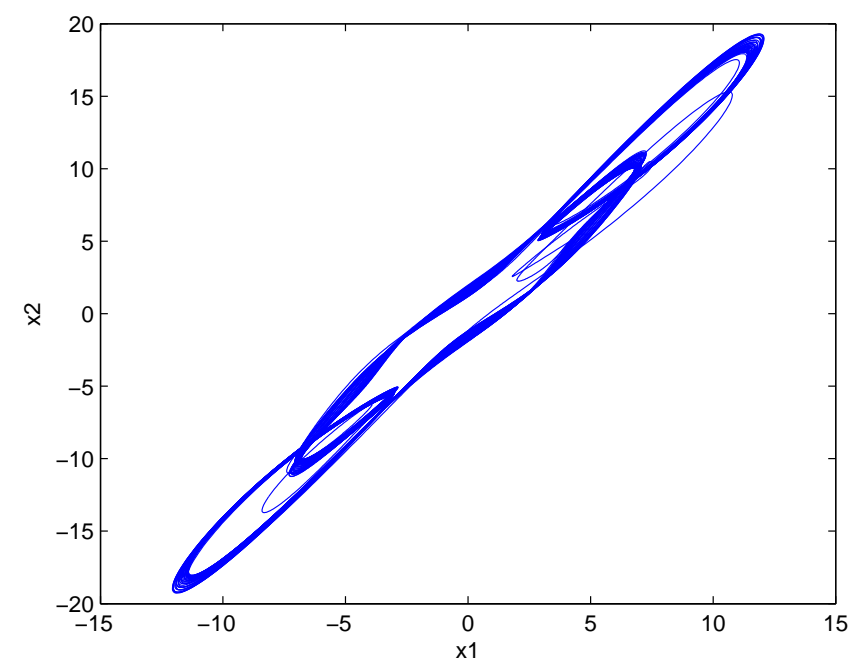

(a)

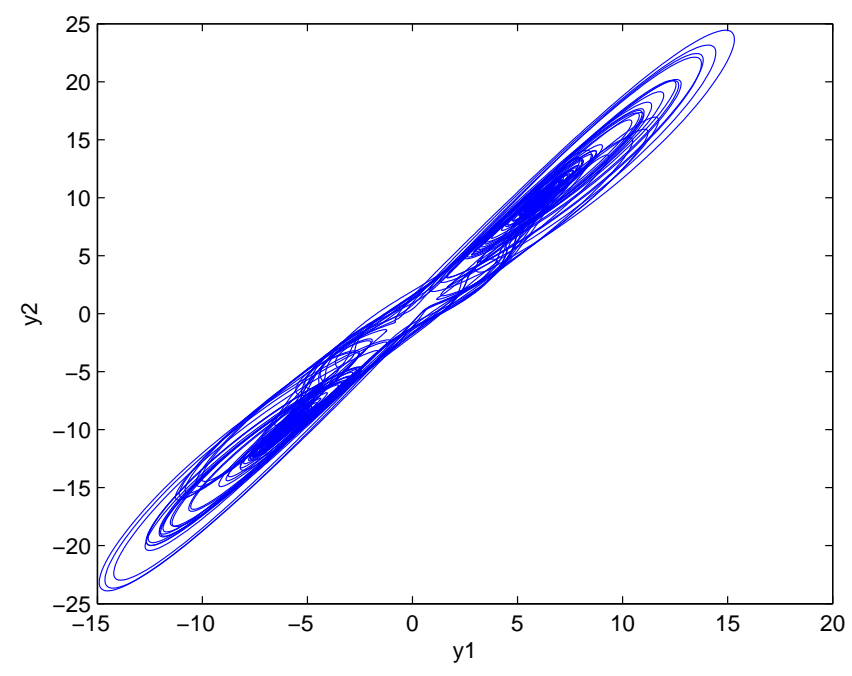

(b)

Fig. 12. Simulation results of the coupled cell system (8), for $a=35.0, b=3.0, c=21$ and $\alpha=0.9$. The cells in the 3-ring appear to show a periodic orbit of "long" period, and the cells in the 5-ring are at a quasiperiodic state. Phase space plot of the first Chen oscillator of (a) 3-ring and (b) 5-ring.

In Fig. 1 [Pinto, 2014], a similar integer order network is considered, with the internal dynamics of each cell modeled by the Chen oscillator. There, these three types of bifurcations occur for increasing values of $c$, analogously to what happens for the fractional order coupled cell system (8). We believe a similar bifurcation diagram must apply here when varying the parameter $c$. In addition, the two-parameter ( $c$ versus $\alpha$ ) bifurcation

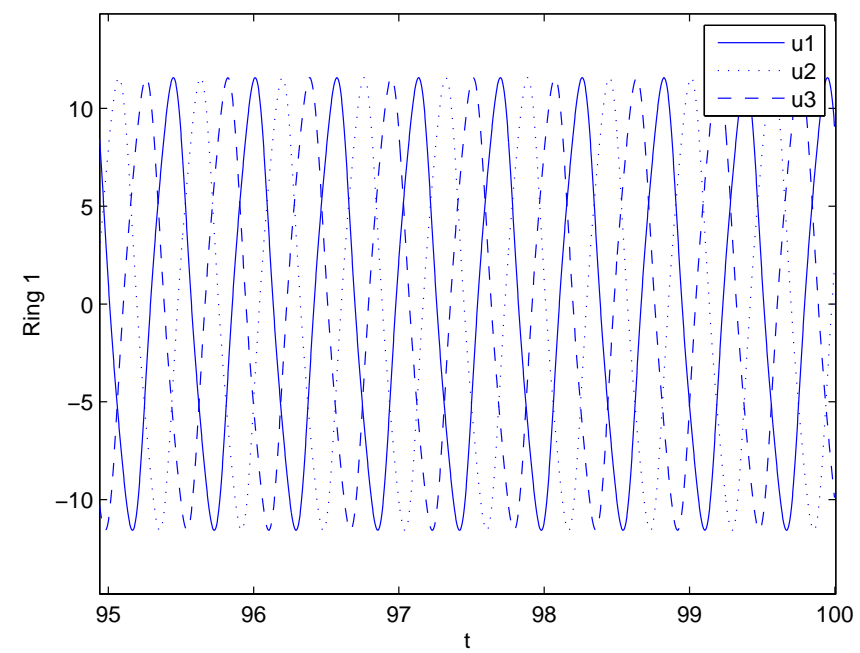

(a) diagram, must add even more complexity to the one-parameter diagram, since distinct features appear for distinct values of $\alpha$. This must be studied in detail in future works.

(v) The presence of symmetry constrains the dynamical behavior of the cells in each of the rings (remember the rotating wave states). Nonetheless, the chaoticity of some solutions seems to be strongly dependant on the choice of the vector field. In this paper, we have

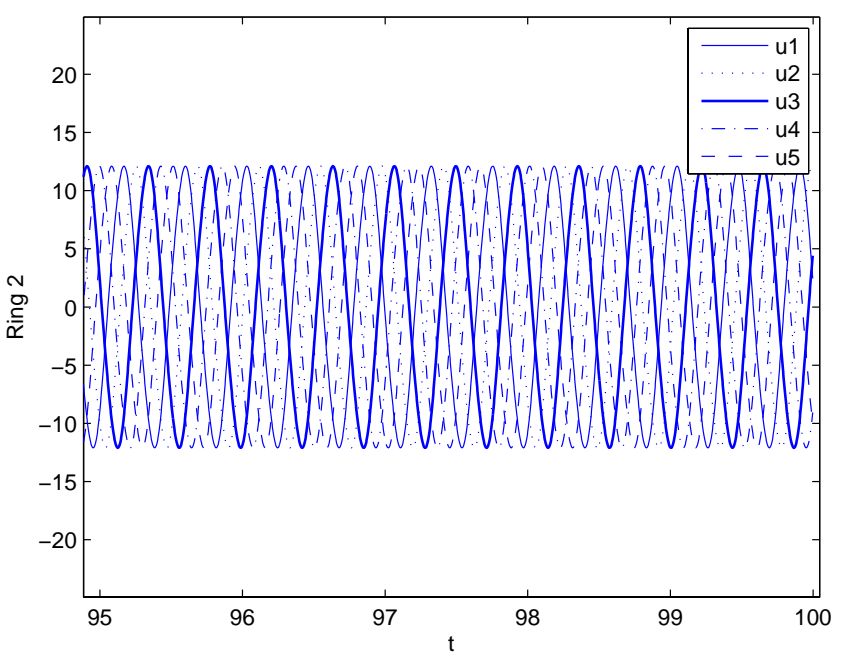

(b)

Fig. 13. Simulation results of the coupled cell system (8), for $a=35.0, b=3.0, c=24$ and $\alpha=1$. The cells in the two rings depict a rotating wave state. For more information, see text. Time series of the first variable $u$ of each Chen oscillator of (a) 3-ring and (b) 5-ring. 


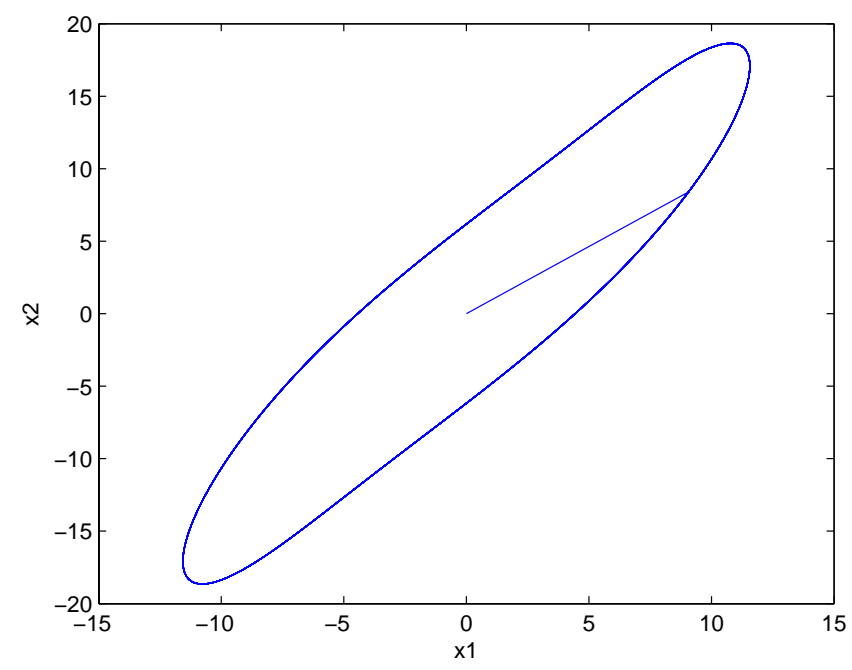

(a)

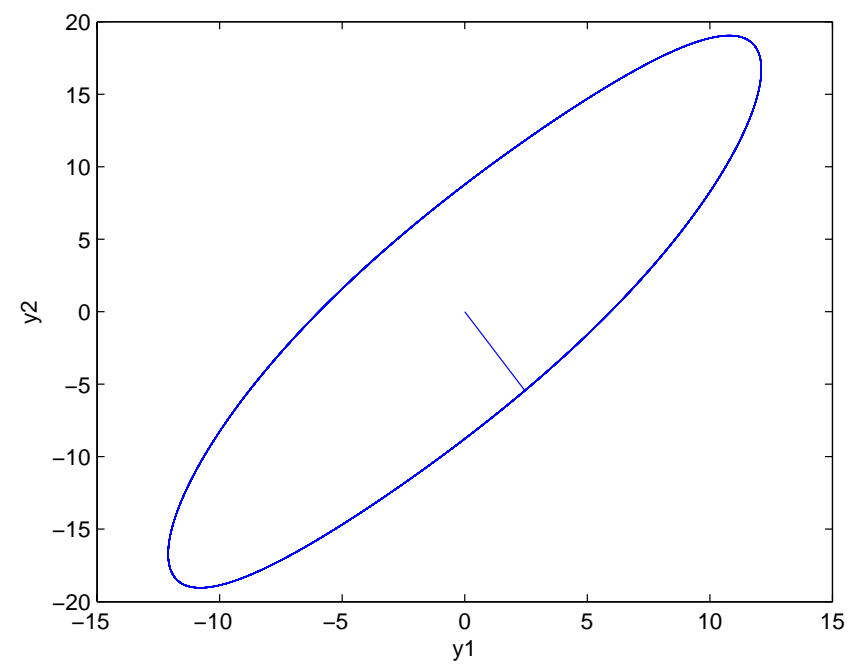

(b)

Fig. 14. Simulation results of the coupled cell system (8), for $a=35.0, b=3.0, c=24$ and $\alpha=1.0$. The cells in the two rings depict a rotating wave state. Phase space plot of the first Chen oscillator of (a) 3-ring and (b) 5-ring.

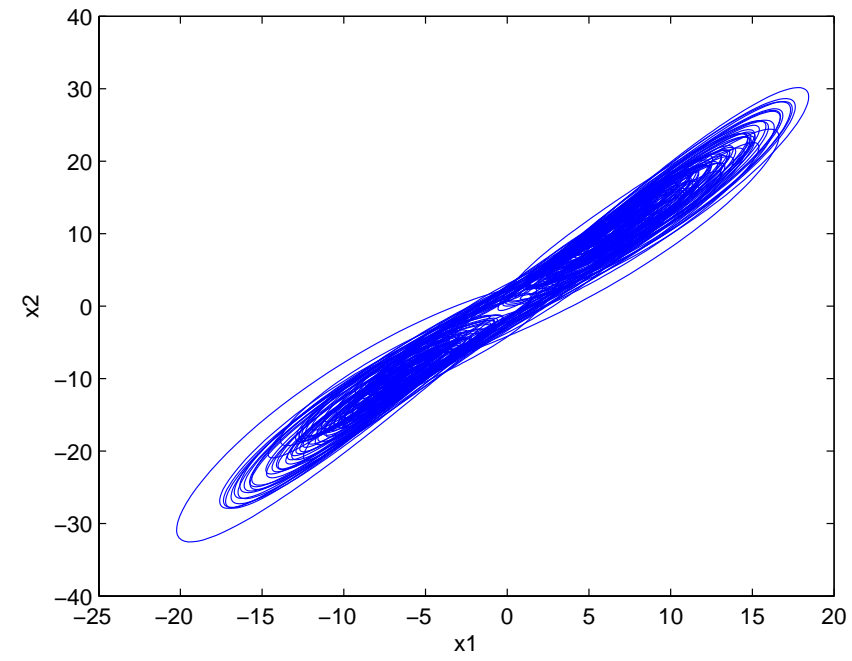

(a)

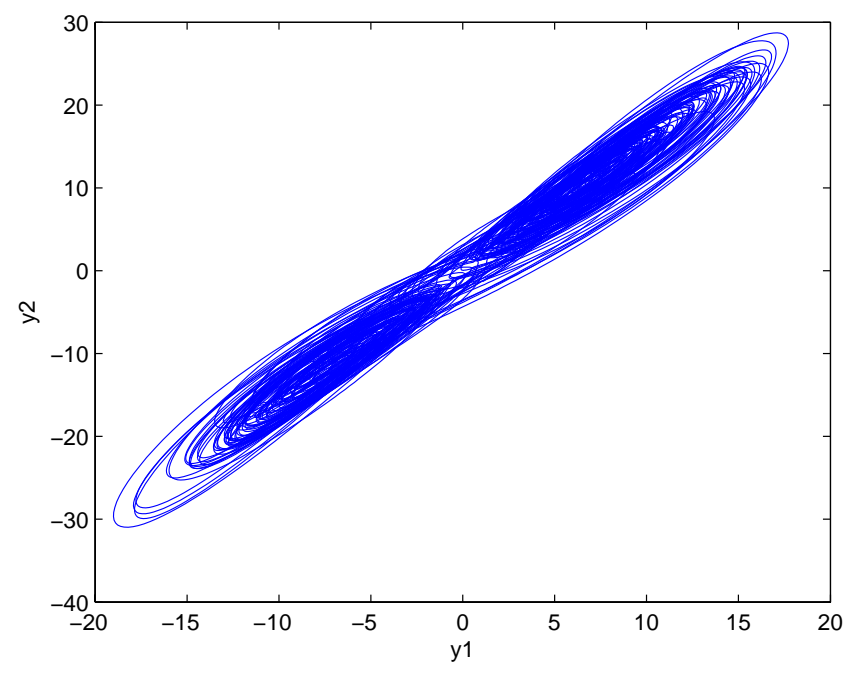

(b)

Fig. 15. Simulation results of the coupled cell system (8), for $a=35.0, b=3.0, c=24$ and $\alpha=0.9$. The cells in the two rings exhibit chaotic motions. Phase space plot of the first Chen oscillator of (a) 3-ring and (b) 5-ring.

considered the Chen oscillator to model the internal dynamics of each node (or cell). This system may exhibit quasiperiodic motion and chaotic states, interleaved with windows of periodic solutions, for variation of the parameter $c$ [Lü et al., 2002].

(vi) From the observation of the figures, one can infer that a way of controlling chaos could rely on the variation of the parameter $c$ and/or the variation of the value of $\alpha$.

(vii) To our knowledge, the theory of Hopf bifurcation in fractional equivariant dynamical systems is far from being developed. This might be an interesting future research topic.

The network seems to "hide" very interesting dynamical patterns and an intriguing bifurcation diagram that will be the focus of future research work.

\section{Conclusion}

Exotic and intriguing patterns of a fractional derivative of complex-order coupled cell system are 
exposed in this work. The coupled cell system is associated with a network of two unidirectional rings of cells, coupled through a "buffer" cell, and has $\mathbf{Z}_{3} \times \mathbf{Z}_{5}$ symmetry group.

Some patterns are indeed expected, due to the network architecture, nevertheless the full comprehension of the behavior of the coupled cell system is only achieved by the inclusion of the internal dynamics of each cell. The internal dynamics may, without a doubt, add more complexity to the "expected" behavioral features of networks of coupled cells. This is verified in this work, where we considered the Chen oscillator as the internal dynamics of the cells in the rings. The order of the fractional derivative also appears to have a significant role in the features produced by the coupled cell system. The latter induces a way of controlling chaos. Future work is needed to explore these findings further.

\section{Acknowledgments}

The author wishes to thank the Polytechnic of Porto, through the PAPRE Programa de Apoio à Publicação em Revistas Científicas de Elevada Qualidade for financial support. The author was partially funded by the European Regional Development Fund through the program COMPETE and by the Portuguese Government through the FCT Fundação para a Ciência e a Tecnologia - under the project PEst-C/MAT/UI0144/2013.

We would like to thank the anonymous reviewers for their valuable suggestions that have very much contributed to the improvement of the quality of our paper.

\section{References}

Aguiar, M. A. D., Ashwin, P., Dias, A. \& Field, M. [2011] "Dynamics of coupled cell networks: Synchrony, heteroclinic cycles and inflation," J. Nonlin. Sci. 21, 271-323.

Albert, R. \& Barabási, A.-L. [2002] "Statistical mechanics of complex networks," Rev. Mod. Phys. 74, 47-97.

Antoneli, F., Dias, A. P. S. \& Pinto, C. M. A. [2010] "Quasi-periodic states in coupled rings of cells," Commun. Nonlin. Sci. Numer. Simul. 15, 1048-1062.

Boccaletti, S., Latora, V., Moreno, Y., Chavez, M. \& Hwang, D.-H. [2006] "Complex networks: Structure and dynamics," Phys. Rep. 424, 175-308.
Chen, G. \& Ueta, T. [1999] "Yet another chaotic attractor," Int. J. Bifurcation and Chaos 9, 1465-1466.

Dachselt, F. \& Schwarz, W. [2001] "Chaos and cryptography," IEEE Trans. Circuits Syst.-I: Fund. Th. Appl. 48, 1498-1509.

Golubitsky, M., Stewart, I. \& Schaeffer, D. G. [1988] Singularities and Groups in Bifurcation Theory, Vol. 2, Applied Mathematical Sciences, Vol. 69 (SpringerVerlag, NY).

Golubitsky, M. \& Stewart, I. [2002] The Symmetry Perspective: From Equilibrium to Chaos in Phase Space and Physical Space, Progress in Mathematics, Vol. 200 (Birkhäuser, Basel).

Golubitsky, M., Nicol, M. \& Stewart, I. [2004] "Some curious phenomena in coupled cell networks," J. Nonlin. Sci. 14, 119-236.

Golubitsky, M., Stewart, I. \& Török, A. [2005] "Patterns of synchrony in coupled cell networks with multiple arrows," SIAM J. Appl. Dyn. Syst. 4, 78-100.

Golubitsky, M. \& Stewart, I. [2006] "Nonlinear dynamics of networks: The groupoid formalism," Bull. Amer. Math. Soc. 43, 305-364.

Hartley, T. T., Adams, J. L. \& Lorenzo, C. F. [2005] "Complex-order distributions," Proc. ASME Int. Design Engineering Technical Conf. Computers and Information in Engineering Conf., Long Beach, CA, DETC2005-84952, on CD-ROM.

Huxter, J., Burgess, N. \& OḰeefe, J. [2003] "Independent rate and temporal coding in hippocampal pyramidal cells," Nature 425, 828-832.

Ikegaya, Y., Aaron, G., Cossart, R., Aronov, D., Lampl, I., Ferster, D. \& Yuste, R. [2004] "Synfire chains and cortical songs: Temporal modules of cortical activity," Science 304, 559-564.

Kilbas, A. A., Srivastava, H. M. \& Trujillo, J. J. [2006] Theory and Applications of Fractional Differential Equations, North-Holland Mathematics Studies, Vol. 204 (Elsevier, Amsterdam).

Krupa, M. \& Szmolyan, P. [2001] "Relaxation oscillations and canard explosion," J. Diff. Eqs. 174, 312368.

Li, C. P. \& Ma, Y. T. [2013] "Fractional dynamical system and its linearization theorem," Nonlin. Dyn. 71, $621-633$.

Lü, J. H., Zhou, T. S., Chen, G. R. \& Zhang, S. C. [2002] "Local bifurcation of the Chen system," Int. J. Bifurcation and Chaos 12, 2257-2270.

Mainardi, F. [2010] Fractional Calculus and Waves in Linear Viscoelasticity: An Introduction to Mathematical Models (Imperial College Press, London).

Miller, K. S. \& Ross, B. [1993] An Introduction to the Fractional Calculus and Fractional Differential Equations (John Wiley \& Sons).

Oldham, K. B. [2010] "Fractional differential equations in electrochemistry," Adv. Eng. Softw. 41, 9-12. 
Pikovsky, A., Rosenblum, M. \& Kurths, J. [2003] Synchronization: A Universal Concept in Nonlinear Science (Cambridge University Press, Cambridge).

Pinto, C. M. A. \& Golubitsky, M. [2006] "Central pattern generators for bipedal locomotion," J. Math. Biol. 53, 474-489.

Pinto, C. M. A. \& Tenreiro Machado, J. A. [2011a] "Complex order biped rhythms," Int. J. Bifurcation and Chaos 21, 3053-3061.

Pinto, C. M. A. \& Tenreiro Machado, J. A. [2011b] "Complex order van der Pol oscillator," Nonlin. Dyn. 65, 247-254.

Pinto, C. M. A. [2012] "Exotic dynamics in networks of coupled rings of cells," Int. J. Bifurcation and Chaos 22, 1250064-1-13.

Pinto, C. M. A. [2014] "Exciting dynamical behavior in a network of two coupled rings of Chen oscillators," Nonlin. Dyn. 78, 1245-1259.

Pinto, C. M. A. \& Tenreiro Machado, J. A. [2014] "Fractional dynamics of computer virus propagation," Mathematical Problems in Engineering, 476502-1-7.

Podlubny, I. [1998] Fractional Differential Equations: An Introduction to Fractional Derivatives, Fractional Differential Equations, to Methods of Their Solution, Mathematics in Science and Engineering, Vol. 198 (Academic Press, San Diego).

Prinz, A. A., Bucher, D. \& Marder, E. [2004] "Similar network activity from disparate circuit parameters," Nat. Neurosci. 7, 1345-1352.

Stewart, I., Golubitsky, M. \& Pivato, M. [2003] "Symmetry groupoids and patterns of synchrony in coupled cell networks," SIAM J. Appl. Dyn. Syst. 2, 609-646.
Stewart, I. \& Parker, M. [2007] "Periodic dynamics of coupled cell networks: I. Rigid patterns of synchrony and phase relations," Dyn. Syst. 22, 389-450.

Stewart, I. \& Parker, M. [2008] "Periodic dynamics of coupled cell networks: II. Cyclic symmetry," Dyn. Syst. 23, 17-41.

Singer, W. [1999] "Neuronal synchrony: A versatile code for the definition of relations," Neuron 24, 49-65.

Tarasov, V. E. [2010] Fractional Dynamics: Applications of Fractional Calculus to Dynamics of Particles, Fields and Media (Springer, NY).

Tenreiro Machado, J. A. [1997] "Analysis and design of fractional-order digital control systems," Syst. Anal. Model. Simul. 27, 107-122.

Terman, D., Rubin, J. E. \& Wilson, C. H. [2002] "Activity patterns in a model for the subthalamopallidal network of the basal ganglia," J. Neurosci. 22, 29632976.

Vragović, I., Louis, E., Degli Esposti Boschi, C. \& Ortega, G. [2006] "Diversity-induced synchronized oscillations in close-to-threshold excitable elements arranged on regular networks: Effects of network topology," Physica D 219, 111-119.

Wang, X. F. [2002] "Complex networks: Topology, dynamics and synchronization," Int. J. Bifurcation and Chaos 12, 885-916.

Xie, Q., Chen, G. \& Bollt, E. M. [2002] "Hybrid chaos synchronization and its application in information processing," Math. Comput. Model. 35, 145-163.

Zhu, J., Lü, J. \& Yu, X. [2013] "Flocking of multi-agent non-holonomic systems with proximity graphs," IEEE Trans. Circuits Syst.-I 60, 199-210. 